Physics International 3 (1): 28-43, 2012

ISSN 1948-9803

(C) 2012 Science Publications

\title{
The Bidispersed Model System and Binary System for Ferrofluids
}

\author{
${ }^{1} \mathrm{Jian} \mathrm{Li}$ and ${ }^{2}$ Decai Li \\ ${ }^{1}$ School of Physical Science and Technology, \\ Southwest University, Chongqing 400715, China \\ ${ }^{2}$ School of Mechanical and Control Engineering, \\ Beijing Jiaotong University, Beijing 100044, China
}

\begin{abstract}
Ferrofluids, dispersions of magnetic nanoparticles, are typical dipolar fluids whose behaviors can be modified using an external magnetic field. Such magnetically controlled effects result from interactions between the magnetic particles, which are enhanced by an applied magnetic field. Since the system of particles always possesses a size distribution, real ferrofluids represent a polydispersed system. A theoretical model of a bidispersed system, which contains only particles of two sizes, large and small, is presented. Experimentally, some binary ferrofluids based on two types of nanoparticles, with different magnetization or containing a mixture of micron-sized particles doped in ferrofluids are investigated. These magnetic colloidal systems have two dispersion phases in which at least one is magnetic nanoparticles and can exhibit novel behavior. This may result in novel physical properties and new potential applications. A model of a bidispersed system based on aggregated and non-aggregated particles, in which some non-aggregated particles could transform into aggregated particles with increasing magnetic field while total particles' number is constant, may help to advance general theories of ferrofluids.
\end{abstract}

Keywords: Ferrofluids, Interaction, Dipole, Particles

\section{INTRODUCTION}

Generally, magnetic (ferromagneitc or ferrimagnetic) materials are used in their solid state, i.e., in a polycrystalline or a single crystal form as bulk materials or thin film deposited on substrates. However, the physical properties and applications of liquid magnetic materials are of great interest. In particular, the question arises as to whether there are any natural magnetic materials in the liquid state. From solid state physics it can be known that although a conventional magnetic material becomes liquid above its melting point, this does not produce a magnetic liquid since the melting point of a ferromagnetic/ferrimagnetic material is usually higher than its Curie point, above which the magnetic material becomes paramagnetic. Consequently, magnetic materials like iron, cobalt, nickel, lose their magnetic properties at temperatures far below their melting points. Liquid metals and other molecular liquids, e.g., paramagnetic salt solutions, require extremely high magnetic fields, of the order of several Teslas, to have any magnetic influence on their behavior (Odenbach, 2003). However, there does exist another class of magnetic field responsive fluids, including Magnetorheological (MR) and ferrofluids, which are colloidal dispersions of finely divided magnetic particles in a appropriate carrier liquid. MR fluids are dispersions that comprise mesocale (1 10 $\mu \mathrm{m})$ ferromagnetic or ferrimagnetic particles dispersed in an organic or aqueous carrier liquid (Phulé, 2001). Ferrofluids (magnetic liquids, ferrocolloids,) are stable colloidal suspensions of monodomain ferromagnetic/ferrimagnetic nanoparticles (about $10 \mathrm{~nm}$ ), dispersed in a carrier liquid. Such nanoparticles contain only a single magnetic domain and can thus be treated as small thermally agitated permanent magnets in a carrier liquid (Holm and Weis, 2005). Ferrofluids are magnetically functional materials with fluidity and some of their properties, such as the microstructure, viscosity or optical behavior, can be changed by applying an external magnetic field. In particular, since they are liquid materials, the field response of ferrofluids produces no residual effects. Therefore, ferrofluids have potentially a wide range of interesting technical and biomedical applications (Buske, 1994; Teixeira et al., 2000).

Corresponding Author: Jian Li, School of Physical Science and Technology, Southwest University, Chongqing 400715, China 
The small size of particles, about $10 \mathrm{~nm}$ in diameter, result in intensive Brownian movement so that a high sedimentation stability is obtained for ferrofluids. In order to prevent the coalescence of the magnetic nanoparticles under the action of van der Waals forces or interaction between magnetic moments or both, the particles are usually coated with surfactants or polymers whose entropic repulsion counteracts the van der Waals forces, or with charged groups that prevent agglomeration due to their electrostatic repulsion.

Ferrofluid particles are too small for the creation of domain walls. For this reason, the quantum-mechanical exchange interactions between the electronic spins allows all the atomic magnetic moments to be aligned so that the total magnetic moment $\mathrm{m}$, of the particles is of the order of about $10^{4}$ Bohr magnetons $\left(\mu_{\mathrm{B}}\right)$. The magnetization $\mathrm{M}$ of ferrofluids can be described by standard paramagnetic behavior but, in contrast to paramagnetic salt solutions, the magnetic units interacting with the magnetic field are not single molecular magnetic moments but particles containing approximately $10^{4} \mu_{\mathrm{B}}$. The ferrofluid particles are so small that they can be regarded as magnetic dipoles with moments $\mathrm{m}\left(=\mathrm{VM}_{\mathrm{s}}\right.$, here $\mathrm{V}$ and $\mathrm{M}_{\mathrm{s}}$ are the volume and saturation magnetization of the particles, respectively). Therefore, ferrofluids can also be viewed as typical magnetic dipolar fluids (Teixeira et al., 2000).

Polydispersity is inherent in all colloid dispersions and needs to be considered in the interpretation of experimental data for ferrofluids, as it affects the magnetic properties (Holm and Weis, 2005). The wellknown difficulties encountered in the statistical physics of systems with interactions hinders the formulation of a rigorous theory of ferrofluids containing even a single type of linear aggregate. As a first approximation, a theory modeled as a bidisperse system, consisting of "large" and "small" particles with different volume fractions, has been proposed to represent a real polydisperse system with a distribution of particle sizes (Zubarev and Iskakova, 2003). In addition, binary ferrofluids based on two types of nanoparticles, with different magnetizations, or micron-sized particles doped in ferrofluids, can be investigated experimentally. Novel behavior is sometimes revealed. This review is organized as follows. The magnetic interaction, which results in field-induced properties for ferrofluids, is presented in 1.1. The essentials of the bidispersion model and its application to experimental results are given in 1.2. The binary ferrofluids, based on two types of magnetic nanoparticles, are described with some experimental data in 1.3. Some novel experimental results are presented for binary ferrofluids based on both micron- and nano-sized particles in 1.4. The review ends with conclusion. The SI units are adopted in the review.

The magnetic interactions in ferrofluids: A remarkable feature of ferrofluids is their ability to change their macroscopic behavior under the influence of an external magnetic field, producing a range of magneto-optical, magneto-viscous and other effects. Such behavior is mainly determined by their magnetic properties. For ferrofluids, the magnetic particles are so small that they can be regarded as simple magnetic dipoles $\mathrm{p}_{\mathrm{m}}$ with a point dipole moment at the center, such that $\mathrm{p}_{\mathrm{m}}=\mu_{0} \mathrm{~m}$, where $\mu_{0}$ is the magnetic permeability in vacuum and $\mathrm{m}$ is the magnetic moment fixed inside the particle. The ferrofluid can be described as a system of particles, $\mathrm{i}=1, \ldots \ldots \mathrm{N}$, each present as a dipole $\mathrm{p}_{\mathrm{mi}}$ carrying a magnetic moment $\mathrm{m}_{\mathrm{i}}$ (Huke and Lucke, 2004; Li et al., 2012), that interact with each other in a pairwise manner via dipole-dipole interaction potentials $\mathrm{U}_{\mathrm{d}-\mathrm{d}}$, which can be written as:

$\mathrm{U}_{\mathrm{d}-\mathrm{d}}=-\frac{\mu_{\mathrm{r}}\left[3\left(\mathbf{p}_{\mathrm{mi}}, \mathbf{r}_{\mathrm{ij}}\right) / \mathrm{r}_{\mathrm{ij}}-\mathbf{P}_{\mathrm{mi}} \cdot \mathbf{P}_{\mathrm{mj}}\right]}{4 \pi \mu_{0} \mathrm{r}_{\mathrm{ij}}^{3}}$

where, $\mu_{\mathrm{r}}$ is the relative magnetic permeability in the magnetic medium and is taken as unity in magnetically neutral medium. $r_{i j}$ is the distance vector between particles $i$ and $j$ (as shown in Fig. 1).

Since this potential decreases only as $\mathrm{r}^{-3}$, the dipolar interaction is long range. Formula (1) shows that for ferrofluids, unlike the exchange interaction with a constant sign, the interaction can change a sign from attraction to repulsion, so is neither always attractive nor always repulsive. As a consequence, the spontaneous magnetization predicted by the Weiss model is unlikely to appear in ferrofluids and has never been observed in any experiment (Holm et al., 2006). Ferrofluid particles are generally spherical. From formula (1), it can be seen that the maximum magnetic interaction potential of two spherical particles in contact with each other head to tail is $U_{d}$. ${ }_{\mathrm{d}, \max }=-\mu_{\mathrm{r}} \mathrm{p}_{\mathrm{mi}} \mathrm{p}_{\mathrm{mj}} / 2 \pi \mathrm{d}_{\mathrm{ij}}{ }^{3}$. Here, $\mathrm{d}_{\mathrm{ij}}=\left(\mathrm{d}_{\mathrm{i}}+\mathrm{d}_{\mathrm{j}}\right) / 2$ and $\mathrm{d}_{\mathrm{i}}$ and $\mathrm{d}_{\mathrm{j}}$ are the diameters of the particles $i$ and $j$, respectively. 


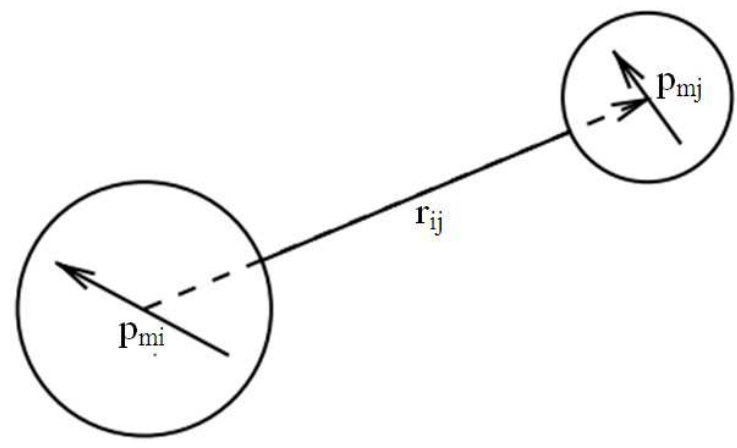

Fig. 1: Spheres $\mathrm{i}$ and $\mathrm{j}$ with magnetic dipoles $\mathrm{p}_{\mathrm{mi}}$ and $\mathrm{p}_{\mathrm{mj}}$, respectively, that interact via the potential

The high fluidity of ferrofluids is combined with a perceptible ability to interact with an external magnetic field, which is the essence of their practical application. Under an external magnetic field $\mathrm{H}$, the magnetic dipole moment $\mathrm{m}$ fixed inside the ferrofluid particles interacts with the field via the potential:

$\mathrm{U}_{\mathrm{m}-\mathrm{H}}=-\mu_{0} \mathbf{m} \cdot \mathbf{H}$

so that the moments tend to line up with the direction of the field. The increase of orientation of the magnetic moment of the particles under the influence of the magnetic field leads to an increase of the effective attraction between the individual ferrofluid particles (Zubarev, 2002). The average degree of alignment of the moments depends on the ratio of $\mu_{0} \mathrm{mH}$ to the thermal energy $\mathrm{k}_{\mathrm{B}} \mathrm{T}\left(\mathrm{k}_{\mathrm{B}}\right.$ is the Boltzmann constant and $\mathrm{T}$ is the absolute temperature), because thermal motion tends to destroy the alignment.

For ferrofluids, the field-induced effects result from the microstructural transitions that take place under the influence of an applied magnetic field. To make allowance for the presence of different magnetic materials and a range of particle sizes significantly complicates the problem of determining the internal microstructure of the ferrofluids. To simplify the maximum possible extent and to obtain physical results in a visual form, convenient for physical analysis, the ferrofluid is treated as a monodispersed suspension of identical single-domain magnetic spheres. When the dipolar interaction is sufficiently strong, ferrofluids exhibit a rich phase behavior as a function of the volume fraction of the particles and external magnetic field. The features of the monodispersed ferrofluid system can be characterized by two dimensionless parameters, the dipole coupling parameter $\lambda$ and the volume fraction of particles $\phi$.
The coupling parameter $\lambda$, which expresses the strength of the interaction between the magnetic particles, is defined as the ratio of the maximum attraction potential in a magnetically neutral medium $\left(\mu_{\mathrm{r}}=1\right)$ between the dipoles $\mathrm{U}_{\mathrm{d}-\mathrm{d} \text {,max }}$ and the thermal energy $\mathrm{k}_{\mathrm{B}} \mathrm{T}$ :

$$
\lambda=-\frac{1}{2} \frac{\mathrm{U}_{\mathrm{d}-\mathrm{d}, \max }}{\mathrm{k}_{\mathrm{B}} \mathrm{T}}=\frac{\mu_{0} \mathrm{~m}^{2}}{4 \pi \mathrm{d}^{3} \mathrm{k}_{\mathrm{B}} \mathrm{T}}
$$

where, the coefficient $1 / 2$ is applied since the coupling parameter is related to a pair of particles. $\lambda$ is also called the coupling constant and is one of the most important parameters used to characterize ferrofluids at $\mathrm{H}=0$. When $\lambda>1$, aggregation of the particles can take place and this is classified as the strong coupling limit (Tavers et al., 1999; Wang et al., 2002). Although the microstructure of a particular fluid is hard to observe experimentally (Teixeira et al., 2000), Klokkenbury and co-worker have revealed chain-like and ring-like structures in ferrofluids at zero field and in an applied magnetic field using Monte Carlo simulation and by direct observation in situ using cryogenic Transmission Electron Microscopy (cryoTEM) (Butter et al., 2003; Klokkenburg et al., 2004; 2006). Employing the Monte Carlo method, Aoshima and Satoh found that for a very weak magnetic field, the strong interaction between the larger particles gives rise to the formation of various shapes from the chainlike clusters, including bending, looping and branching; in a strong magnetic field, chain-like clusters are formed along the magnetic field direction (Aoshima and Satoh, 2005). For $\lambda<1$, the system is regarded as being in a homogeneous "gas-like" state with insufficient magnetic interaction to make the particles aggregate (Psheichnikor and Mekhonoshin, 2000).

The volume fraction of the particles $\phi$, which is another dimensionless parameter used to characterize ferrofluids, is defined as:

$$
\begin{aligned}
\phi & =\frac{\text { volume of the particles }}{\text { volume of the particles }+ \text { volume of the carrier liquid }} \\
& =\frac{\mathrm{N}}{\mathrm{V}} \frac{\pi \mathrm{d}^{3}}{6}
\end{aligned}
$$

where, $\mathrm{N}$ is the number of particles in the ferrofluid, $\mathrm{V}$ is the volume of the ferrofluid and $\mathrm{N} / \mathrm{V}$ denotes the number density of the particles. In ordinary ferrofluids, $\phi$ is typically a few percent (Odenbach, 2003; Huke and Lucke, 2004). For 
ferrofluids, the interparticle interaction is related to $\phi$ and $\lambda$ (Mérigue et al., 2004). A modern approach to the description of the aggregated chain structure in ferrofluids is based on the free-energy density functional method. According to this, the ferrofluid free energy is expressed as a function of the concentration $g_{n}$ of chains consisting of $n$ particles. Traditionally, for ferrofluids of low concentration, such as $\phi=5 \%$, the interchain interaction is neglected and the interparticle interaction is taken into account only for nearest neighboring particles in each chain (Mendelev and Ivanov, 2005). It is usually considered that for small particle volume fractions $(\phi<1 \%)$, the ferrofluid remains in the dilute limit and the magnetic interactions between ferrofluid particles is negligible and cannot stimulate particle aggregation (Dubois et al., 1999; Hamonay et al., 1999).

Experiments, theories and simulation have shown that for large enough values of $\lambda$ and $\phi$, the ferrofluid particles tend to organize themselves into many different types of aggregates, such as chains, rings, branched structures and networks. In addition, in the non-aggregating regime of $\lambda \sim 1$, despite the fact that the proportion of aggregates is negligible, correlations can still exist between particles from the magnetic interparticle interaction and the ferrofluid can show anisotropic behavior in a magnetic field (Cerdà et al., 2010; Elfimova et al., 2012). The magnetization curves of ferrofluids are significantly influenced by the field-induced aggregation effect (Li et al., 2007a). The previous theoretical model treated the ferrofluids as ideal superparamagnetic materials, in which there are no magnetic interactions between the magnetic colloidal particles. On the other hand, if there is a strong interaction between the magnetic colloidal particles, they will attract each other and no longer be dispersed in the carrier liquid. Neglecting the magnetic interaction between the colloidal particles and taking only the mutual interaction between the $\mathrm{m}$, the magnetic moment of the particles and an applied magnetic field $\mathrm{H}$ into account, the equilibrium magnetization $M$ as $a$ function of $\mathrm{H}$ is expressed by:

$$
\mathrm{M}(\mathrm{H})=\mathrm{M}_{\mathrm{s}} \phi \int_{0}^{\infty} \mathrm{L}(\alpha) \mathrm{f}_{\mathrm{dis}}(\mathrm{V}) \mathrm{dV}
$$

where, $M_{s}$ and $\phi$ are the saturation magnetization and volume fraction of the colloidal particles, respectively, the function $f_{\text {dis }}(V)$ is the normalized particle size distribution function of the particles expressed in terms of their volume $\mathrm{V}, \mathrm{L}(\alpha)=$ cotha- $1 / \alpha$ is the Langevin function. In this expression, $\alpha=\mathrm{M}_{\mathrm{s}} \mathrm{VH} / \mathrm{k}_{\mathrm{B}} \mathrm{T}$ is the Langevin parameter. For spherical particles and not taking into account any distribution of the particle size, formula (5) can be written simply as:

$\mathrm{M}(\mathrm{H})=\mathrm{M}_{\mathrm{s}} \phi \mathrm{L}(\alpha)$

where, $\alpha=\mu_{0} \mathrm{mH} / \mathrm{k}_{\mathrm{B}} \mathrm{T}, \mathrm{m}=\mathrm{M}_{\mathrm{s}} \pi \mathrm{d}^{3} / 6$ and $\mathrm{d}$ is average diameter of the particles. In a low field, $L(\alpha)=\alpha / 3$ and formula (6) leads to Curie's law for the initial susceptibility $\chi_{\mathrm{L}}$ :

$\chi_{\mathrm{L}}=\left.\frac{\partial \mathrm{M}}{\partial \mathrm{H}}\right|_{\mathrm{H} \rightarrow 0}=\frac{\mu_{0} \mathrm{M}_{\mathrm{s}}^{2} \pi \mathrm{d}^{3}}{18 \mathrm{k}_{\mathrm{B}} \mathrm{T}}$

By comparing the shapes of the magnetization curves and initial susceptibilities of ferrofluids with high $\phi$ as mother fluid and those of corresponding diluted solution, the magnetic interactions between ferrofluid particles have been clarified experimentally (Taketomi and Shull, 2002; 2003). It was concluded that a small number of clusters with aligned magnetization vectors do exist in the mother ferrofluid $(\phi \approx 10 \%)$ but not when subject to 1000 fold dilution $((\phi \approx 0.01 \%)$.

Clearly, the magnetic interaction between ferrofluid particles is dependent not only on the magnetization of the particles, but also on the volume fraction of the particles. Experimentally, the interparticle magnetic interaction is evaluated using the parameter $\gamma$ (Mérigue et al., 2004; Ivanov and Kuznetsova, 2001), which is defined as:

$$
\gamma=\frac{\mu_{0} \mathrm{~m}^{2}}{<\mathrm{r}>^{3} \mathrm{k}_{\mathrm{B}} \mathrm{T}}=\mu_{0} \mathrm{M}_{\mathrm{s}} \frac{\pi \mathrm{d}^{3}}{6} \frac{\phi}{\mathrm{k}_{\mathrm{B}} \mathrm{T}}
$$

where, $\langle\mathrm{r}\rangle\left(=\phi^{-1} \pi \mathrm{d}^{3} / 6\right)$ is the mean interparticle distance. While $\gamma \leq 1$, the ferrofluids can be descripted by "mean-field model" by Weiss, in which the initial susceptibility as $\chi=\chi_{L} /\left(1-4 \pi \chi_{L} / 3\right)$, or by "modified mean-field model" in which $\chi=\chi_{\mathrm{L}}$ $\left(1+4 \pi \chi_{\mathrm{L}} / 3\right)$. The Weiss model predicts a magnetic phase transition into a magneto-ordered liquid state; at the transition point the initial susceptibility becomes infinite, that is, $\chi \rightarrow \infty$ when $\chi_{L} \rightarrow 3 / 4 \pi$. But a paramagnetic to ferromagnetic second order phase transition is nover observed in fluidlike magnetic system. That is why the Weiss mean-field theory appears to be questionable while applying to ferrofluids (Ivanov and Kuznetosova, 2001). 
The bidispersed model of ferrofluids: Nanoparticles always have a finite size distribution, so real ferrofluids are polydispersed, that is, polydispersity is a natural property of ferrofluids (Cabuil, 2000). To simplify, a ferrofluid system can usually be viewed as a monodispersed suspension of identical one-domain ferromagnetic spheres. This allows the maximum possible theoretical description and can predict physical results in a visual form convenient for physical analysis (Zubarev and Chirkov, 2010). The monodispersed model of (strictly speaking) polydispersed ferrofluids naturally assume all particles possess a system averaged size, that is, have small dimensions. Therefore, these models cannot provide a satisfactory description of the properties of a polydispersed system, except in the case of highly diluted solutions where no chain aggregates or other heterostructures can appear (Zubarev, 2001). The influence of the particle interaction on the magnetic properties is most evident at a weak field. By means of molecular dynamics simulations, the formation of chains is found to enhance the magnetization at weak fields and thus leads to a larger initial susceptibility (Wang et al., 2002). However, Taketomi and Shull (2002; 2003) found experimentally that the initial susceptibility of a system with such interaction could be less than that without interaction (Taketomi and Shull, 2002). This paradox was explained as due to the formation of closed chains under zero field, which may result from the "depletion force" of the polydispersed ferrofluids. In addition, Monte Carlo simulations show that the thickness of the chainlike clusters for a polydispersed system depend on the standard deviation of the particle size distribution but are independent of the magnetic field strength (Aoshima and Satoh, 2005). Obviously, for true ferrofluids, the monodispersed model is very approximate.

The well-known difficulties encountered in the statistical physics of dense systems hinder the formulation of a rigorous theory of ferrofluids containing even a single type of linear aggregates. A first approximation, modeled by a bidispersed system that consists of "large" and "small" particles with different volume fractions, has been proposed instead of a real polydispersed system with more or less uniform particle size distribution (Zubarev, 2003; 2001). For such a bidispersed system, as a result of the magnetic dipole interaction, the coupling parameter between large particles is greater than 1 and the large particles can combine into aggregates. Also, the coupling parameters between small particles and between small and large particles are smaller than 1 , so that the small particles remain in the individual non-aggregated state. Consequently, the chain and droplet aggregates formed from large particles can be visualized as being in a "sea" or "gas" of small particles. It has been demonstrated that small particles can either prevent or stimulate these structural transformations, depending on the properties of the medium. It is well-known that the presence of the gas of small particles provides the so-called "depletion forces", acting as an effective attraction between large particles and leads, even when there is no direct physical attraction between these particles, to a range of diverse phase transitions. Simple geometric considerations show that, when the large particles are assembled into chains and other clusters, the induced excluded volume of the small particles decreases, as shown in Fig. 2. It is clear that this leads to a decrease of the free energy of the entire system, thus stimulating cluster formation. Therefore, it can be expected that the phase and structural transitions in real polydispersed ferrofluids substantially differ from the predicted by the idealized monodispersed models (Zubarev and Iskakova, 2003).

Since the dipole moments of the particles are proportional to the volume of their magnetic cores, the large particles can be oriented more easily at low field strengths and the smaller ones can only be oriented under a rather strong applied magnetic field. As a result, the magnetization properties of a polydispersed system differ from those of a monodispersed system even in the very dilute case. It has been demonstrated that this small fraction of large particles can play a major role in determining the physical properties of a polydispersed ferrofluid (Wang and Holm, 2003). On the basis of the polydispersed model, the main type of structure in these systems is reckoned to be a chainlike aggregate formed by the large particles. Most of the small particles remain in the non-aggregated state rather than chained to each other. A small number of them stick to the edges of the chain formed by the large particles so that the chains remain short (Kantorovich, 2005). When the total volume fraction of the system is fixed, the average size of these clusters increases with increasing volume fraction of large particles. Fixing the volume fraction of large particles, the average aggregate size formed by them decreases when increasing the volume fraction of small particles (Wang and Holm, 2003). 
Phy. Intl. 3 (1): 28-43, 2012

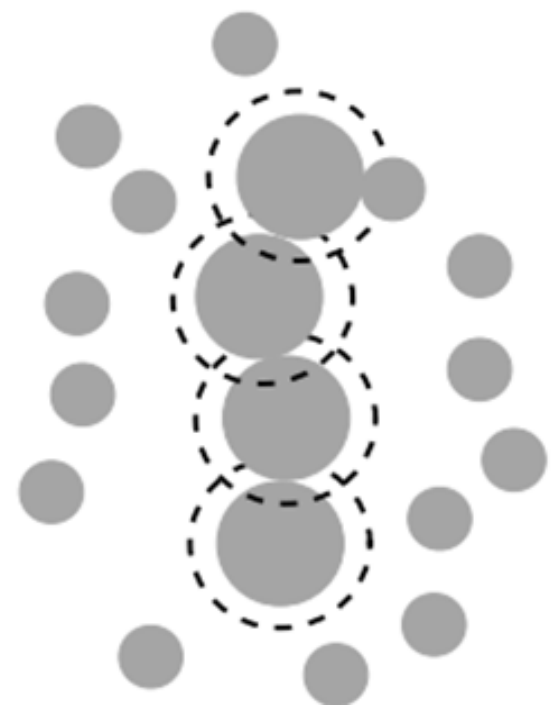

Fig. 2: The determination of the excluded volume for the chain-small particle system (Zubarev and Iskakova, 2003).

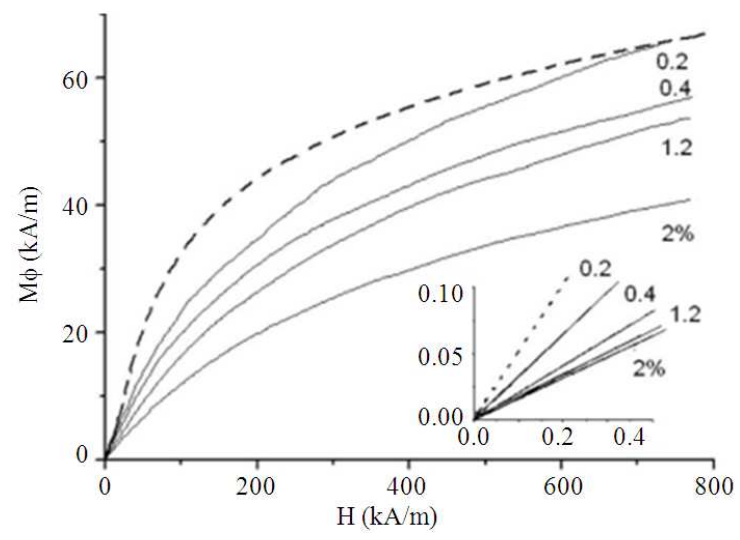

Fig. 3: The magnetization curves reduced by $\phi$ vs applied magnetic field for ferrofluids based on $\mathrm{ZnFe}_{2} \mathrm{O}_{4}$ nanoparticles. The dashed line is the magnetization curve of the particle (Wang et al., 2009)

Ionic $\mathrm{ZnFe}_{2} \mathrm{O}_{4}$ is a weak magnetic ferrofluid with a range of particle sizes (a typical polydispersed ferrofluid), in which the dipolar coupling constant $\lambda$ (= 0.18) is less than 1 (Wang et al., 2009). The magnetization curves were measured and are shown in Fig. 3, scaled to the volume fraction of particles $\phi$. By comparing the reduced magnetization curves and initial magnetic susceptibilities of ferrofluids with different volume fractions of particles, it is revealed that there are field-induced attractive interactions between the colloidal particles, which make them aggregate; this interaction decreases with $\phi$ (Wang et al., 2009). The dipolar coupling constant is so small that particle aggregation cannot be induced by magnetic interaction alone. Thus, it is concluded from the experimental results that a non-magnetic attractive interaction is stimulated during the magnetization process for the $\mathrm{ZnFe}_{2} \mathrm{O}_{4}$ ferrofluids. Using a bidispersed model based on "large" and "small" particles, this non-magnetic interaction can be explained as a "field-induced structure force". In a similar way to the so-called "depletion force" (Birdi, 1997), the field-induced structure force $\mathrm{F}_{\text {str }}$ experienced by one of the larger particles can be written as:

$\mathrm{F}_{\text {str }}=-\mathrm{k}_{\mathrm{B}} \mathrm{T}\left(\mathrm{n}_{2}-\mathrm{n}_{1}\right) \mathrm{S}_{\mathrm{a}}$

where, $S_{a}$ is the effective area between the large particles and $\mathrm{n}_{1}, \mathrm{n}_{2}$ are the number density of small particles inside and outside this area, respectively, as shown in Fig. 4. In zero magnetic field, the colloidal particles act as Brownian particles, so the separation between the particles is random and the large particles can be regarded as being immersed in a "sea" of small particles, as shown in Fig. 4a. When an external magnetic field $\mathrm{H}$ is applied, the magnetic moments inside the particles interact with this magnetic field via the potential $\mathrm{U}_{\mathrm{m}-\mathrm{H}}=-\mu_{0} \mathrm{mH}$. Thus, the moments tend to align with the direction of the field. The magnetic moment $\mathrm{m}$ is proportional to the cube of the particle diameter, so the larger the particle is, the stronger the interaction between the particle and the magnetic field. Therefore, the moments inside the larger particles align more easily to the direction of the applied magnetic field than those inside smaller particles. Also, a weak magnetic attraction interaction could arise between neighboring large particles and force them to approach each other, as shown in Fig. 4b. However, this magnetic interaction is not strong enough to make the particles aggregate since $\mathrm{m}$ is small and the influence of the thermal disturbance is strong. At the same time, as two large particles approach each other, the number density of the smaller particles remaining in the area between the large particles will decrease, as shown in Fig. 4c. Thus, the probability that each large particle is hit by small particles is not isotropic and the probability of an impact from the direction of separation space between the large particles is less than that from other directions. These asymmetric impacts will promote the approach of two large particles, thus giving rise to an attractive force between two large particles in addition to the magnetic attraction. Thus, during the magnetization process the large particles will form aggregates, as shown in Fig. 4d. The additional attractive force between two 
particles is different from the general "depletion force" in an isolated system with no other interactions, because it depends on the attractive magnetic interaction induced by an external magnetic field. As a result, it can be called a "field-induced structural force". This field-induced structural force can also arise in strong magnetic ferrofluids $(\lambda>1)$ with a distribution of particle sizes, which could be difficult to distinguish from the magnetic attraction because this is strong enough by itself to make the particles aggregate. Apparently, when $\mathrm{n}_{1}=0$, formula (9) simply describes the general depletion force (Birdi, 1997).

Experimentally, the interparticle magnetic interaction in a ferrofluid is directly proportional to the volume fraction of the particles $\phi$. For the formation of pre-existing aggregates in zero field, the interaction is regarded as negligible when $\phi$ is less than $1.0 \%$. In the case of non-interacting dipoles, ferrofluids are referred to as "superparamagnetic" liquids, which are characterized by magnetization curves displaying no hysteresis. The magnetization behaviors of polydispersed ferrofluids based on $\gamma-\mathrm{Fe}_{2} \mathrm{O}_{3} / \mathrm{Ni}_{2} \mathrm{O}_{3}$ composite nanoparticles, with coupling constant $\lambda 0.43$, have been investigated ( $\mathrm{Li}$ et al., 2012). The dipole coupling constant of these nanoparticles is too small so that they cannot form aggregates through magnetic interaction alone. Experimental results show that when the particle volume fraction $\phi$ is $2.4 \%$, the magnetization curve exhibits quasi-magnetic-hysteresis behavior, i.e., the demagnetization curve lies above the magnetization in a high field. However, for a more dilute ferrofluid with $\phi=$ $0.94 \%$, the magnetization curve does not exhibit such behavior and gives the results shown in Fig. 5. According to the bidispersed model for polydispersed ferrofluids, this magnetization behavior can be attributed to the fieldinduced effects of self-assembled pre-existing chain-like aggregates, which could originate from a non-magnetic "depletion force". For such pre-existing chain-like aggregates, the orientation of the moments inside the particles is not co-linear, as shown in Fig. 6, so that during the magnetization and demagnetization process, the apparent magnetizations in the high-field limit are different. As a consequence, the magnetic magnetization curve of the ferrofluid with $\phi=2.4 \%$ shows quasimagnetic-hysteresis behavior. When the volume fraction of the particles is below the limit of a dilute ferrofluid (when $\phi=0.94 \%$ ), the apparent magnetization results from the contribution of individual particles, i.e., the particles cannot form aggregates. Therefore, ferrofluids with $\phi=0.94 \%$ do not exhibit hysteresis phenomena similar to those with $\phi=2.4 \%$.

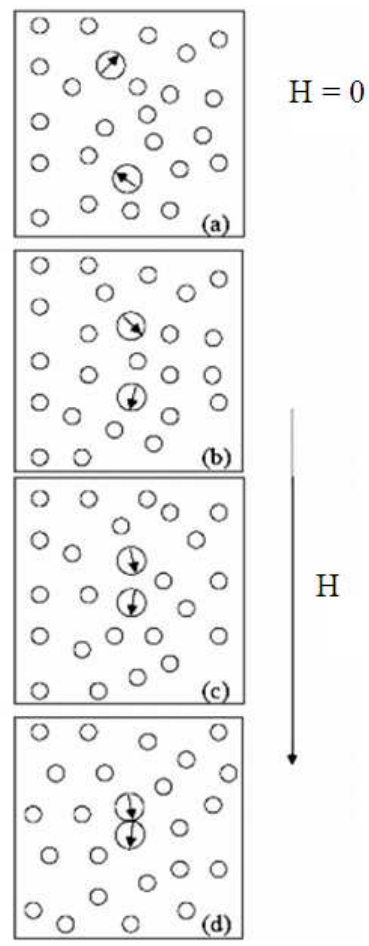

Fig. 4:Schematic illustration of the field-induced structural force between large particles in the bidispersed model for $\mathrm{ZnFe}_{2} \mathrm{O}_{4}$ ferrofluids. Note the depletion of small particles from the space separating large particles as the distance between the large particle decreases and the increase of total magnetic moment of the particle ensemble with applied magnetic field (Wang et al., 2009)

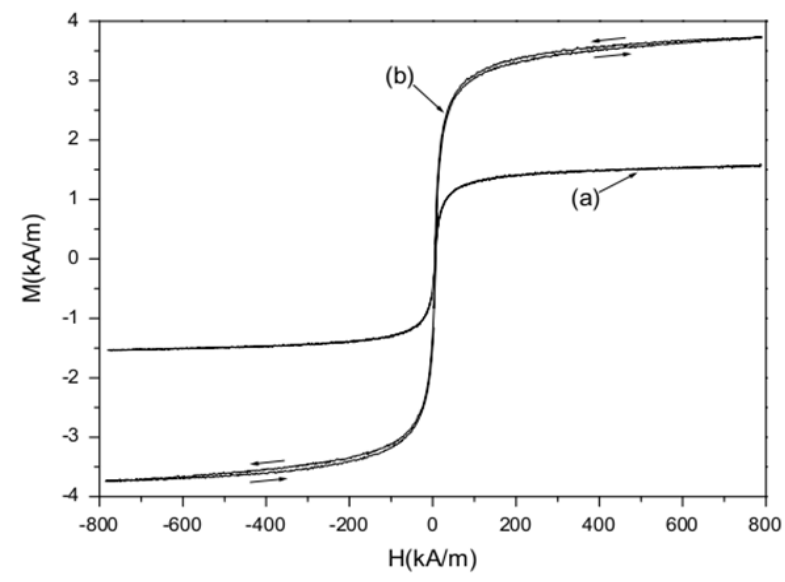

Fig. 5:Magnetization curves of ferrofluids based on $\gamma$ $\mathrm{Fe}_{2} \mathrm{O}_{3} / \mathrm{Ni}_{2} \mathrm{O}_{3}$ composite nanoparticles with (a) $\phi$ $=0.94 \%$ and (b) $\phi=2.4 \%$ (Li et al., 2012) 


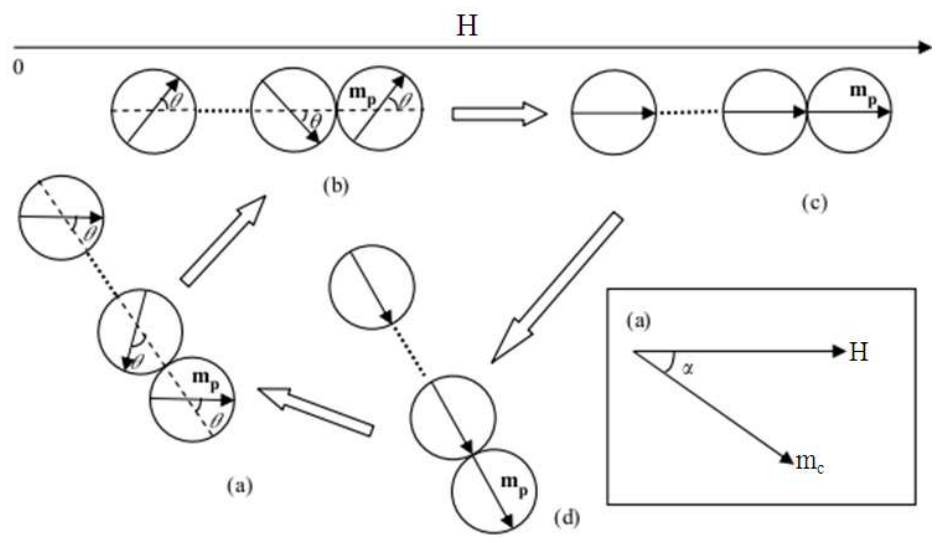

Fig. 6: Geometry of the Model-Of-Chain (MOC) used to characterize the magnetization and demagnetization process for $\gamma-\mathrm{Fe}_{2} \mathrm{O}_{3} / \mathrm{Ni}_{2} \mathrm{O}_{3}$ composite nanoparticle based ferrofluids ( $\mathrm{Li}$ et al., 2012)

The binary ferrofluids based on nano-nano particles: A bidispersed system consisting of large and small particles with no size distribution is hard to achieve experimentally. The magnetic interaction between magnetic nanoparticles is related to the interaction between magnetic moments. The magnetic moment of a single domain particle $\mathrm{m}$ is proportional to its volume $\mathrm{V}$ and, for a spherical particle, can be expressed as:

$$
\mathrm{m}=\pi \mathrm{d}^{3} \mathrm{M} / 6
$$

where, $\mathrm{M}$ is the magnetization of the particle and $\mathrm{d}$ is its diameter. Therefore, the bidispersed modeled ferrofluids consisting of both "large" and "small" particles can be also regarded as a system comprising of "strong" and "weak" magnetic particles, with different $\mathrm{m}$ for different $\mathrm{d}$ ( $\mathrm{Li}$ et al., 2008). Ferromagnetic or ferrimagnetic materials have intrinsic moments. Accordingly, in formula (10), $\mathrm{M}$ is the saturation magnetization for the ferromagnetic/ferrimagnetic nanoparticles. Paramagnetic materials have induced moments along the direction of the applied magnetic field and their magnetization $\mathrm{M}$ is expressed by $\chi \mathrm{H}$, where $\chi$ is the susceptibility and $\mathrm{H}$ is the strength of the applied magnetic field. Accordingly, we propose that the difference in the magnetization between single systems (having different magnetizations $\mathrm{M}$ ) can be employed to synthesise a bidispersed system. That is, one can use a mixture of two pure system with different magnetizations, such as ferrimagnetic and paramagnetic, to produce a magnetically bidspersed system instead of a size bidispersed system. Such fluids based on a mixture of two different magnetic nanoparticles are known as binary ferrofluids and can have behaviors different to those of single ferrofluids (Han et al., 2009; Zhang et al., 2010).

Like general ferrofluids, binary ferrofluids based on A (strongly magnetic) and B (weakly magnetic) nanoparticles, can still be described by both the dipolar coupling constants and volume fraction of particles. For binary ferrofluids, the two dimensionless parameters characterizing ferrofluids, the volume fraction of particles and the coupling constant, are defined as:

$$
\begin{aligned}
& \phi_{\mathrm{AB}}=\frac{\mathrm{V}_{\mathrm{a}}+\mathrm{V}_{\mathrm{b}}}{\mathrm{V}_{\mathrm{a}}+\mathrm{V}_{\mathrm{b}}+\mathrm{V}_{\mathrm{c}}} \\
& \phi_{\mathrm{A}}=\frac{\mathrm{V}_{\mathrm{a}}}{\mathrm{V}_{\mathrm{a}}+\mathrm{V}_{\mathrm{b}}+\mathrm{V}_{\mathrm{c}}} \\
& \phi_{\mathrm{B}}=\frac{\mathrm{V}_{\mathrm{b}}}{\mathrm{V}_{\mathrm{a}}+\mathrm{V}_{\mathrm{b}}+\mathrm{V}_{\mathrm{c}}}
\end{aligned}
$$

where, $\mathrm{V}_{\mathrm{a}}, \mathrm{V}_{\mathrm{b}}$ and $\mathrm{V}_{\mathrm{c}}$ are volumes of the A particles, $\mathrm{B}$ particles and carrier liquid; $\phi_{\mathrm{AB}}$ represents the total volume fraction of both $\mathrm{A}$ and $\mathrm{B}$ particles and $\phi_{\mathrm{A}}, \phi_{\mathrm{B}}$ are the volume fractions of $\mathrm{A}$ and $\mathrm{B}$ particles, respectively. The dipolar coupling constants include those between $\mathrm{A}$ particles $\lambda_{\mathrm{A}-\mathrm{A}}$, between $\mathrm{B}$ particles $\lambda_{\mathrm{B}-\mathrm{B}}$ and between $\mathrm{A}$ and $\mathrm{B}$ particles $\lambda_{\mathrm{A}-\mathrm{B}}$. These are written as:

$$
\begin{aligned}
& \lambda_{\mathrm{A}-\mathrm{A}}=\frac{\mu_{0} \mathrm{~m}_{\mathrm{A}}^{2}}{4 \pi<\mathrm{d}_{\mathrm{A}}>^{3} \mathrm{k}_{\mathrm{B}} \mathrm{T}}, \mathrm{m}_{\mathrm{A}}=\frac{\pi}{6}<\mathrm{d}_{\mathrm{A}}^{3}>\mathrm{M}_{\mathrm{A}} \\
& \lambda_{\mathrm{B}-\mathrm{B}}=\frac{\mu_{0} \mathrm{~m}_{\mathrm{B}}^{2}}{4 \pi<\mathrm{d}_{\mathrm{B}}>^{3} \mathrm{k}_{\mathrm{B}} \mathrm{T}}, \mathrm{m}_{\mathrm{B}}=\frac{\pi}{6}<\mathrm{d}_{\mathrm{B}}^{3}>\mathrm{M}_{\mathrm{B}}
\end{aligned}
$$




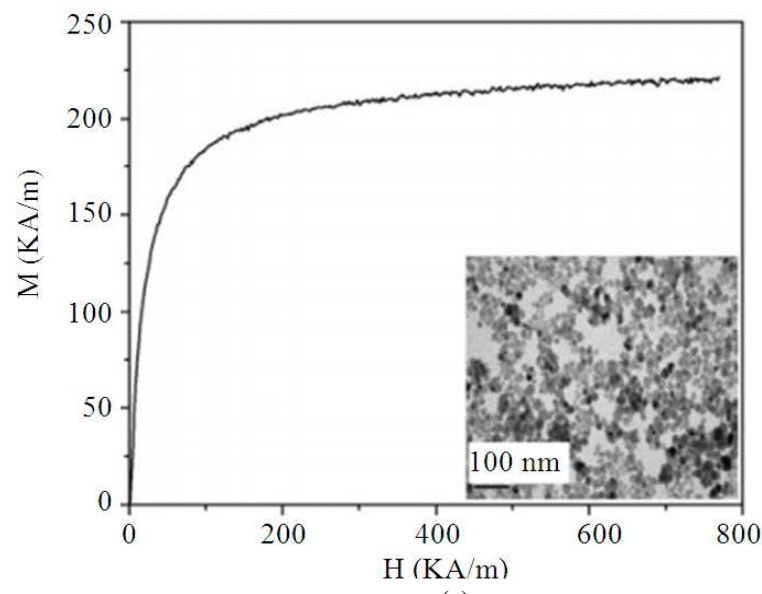

(a)



(b)

Fig. 7: The magnetization curves of (a) $\gamma-\mathrm{Fe}_{2} \mathrm{O}_{3}$ nanoparticle and (b) $\mathrm{ZnFe}_{2} \mathrm{O}_{4}$ nanoparticles. The inserts are the Transmission Electron Microscopy (TEM) photographs of these particles (Fu et al., 2012)

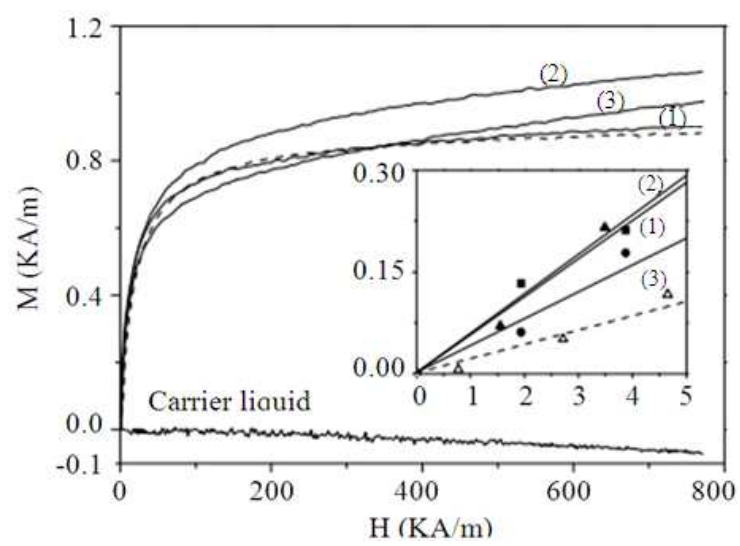

Fig. 8: The magnetization curves of the ferrofluids. $\boldsymbol{\Delta}$, (1): the binary ferrofluids with $\phi_{\gamma}=0.4 \%$ and $\phi_{\mathrm{Zn}}=$ $0.1 \% ; \mathbf{n},-(2)$ : the binary ferrofluids with $\phi_{\gamma}=0.4 \%$ and $\phi_{\mathrm{Zn}}=0.2 \%$;, , -(3): the binary ferrofluids with $\phi \gamma=0.4 \%$ and $\phi_{\mathrm{Zn}}=0.4 \% ; \Delta,-$ : the $\gamma-\mathrm{Fe}_{2} \mathrm{O}_{3}$ nanoparticles multiplied by $0.4 \%$ (Fu et al., 2012)

and:

$$
\begin{aligned}
& \lambda_{\mathrm{A}-\mathrm{B}}=\frac{2 \mu_{0} \mathrm{~m}_{\mathrm{A}} \mathrm{m}_{\mathrm{B}}}{\pi<\mathrm{d}_{\mathrm{A}}+\mathrm{d}_{\mathrm{B}}>^{3} \mathrm{k}_{\mathrm{B}} \mathrm{T}}= \\
& \frac{2 \mu_{0} \mathrm{~m}_{\mathrm{A}} \mathrm{m}_{\mathrm{B}}}{\pi\left(<\mathrm{d}_{\mathrm{A}}>+<\mathrm{d}_{\mathrm{B}}>\right)^{3} \mathrm{k}_{\mathrm{B}} \mathrm{T}}
\end{aligned}
$$

where, $\mathrm{m}_{\mathrm{A}}, \mathrm{m}_{\mathrm{B}}$ and $\left\langle\mathrm{d}_{\mathrm{A}}\right\rangle,\left\langle\mathrm{d}_{\mathrm{B}}\right\rangle$ are the average magnetic moments and the average diameters of the $A$ and $\mathrm{B}$ particles, $\mathrm{M}_{\mathrm{A}}, \mathrm{M}_{\mathrm{B}}$ and $\pi<\mathrm{d}_{\mathrm{A}}{ }^{3}>/ 6, \pi<\mathrm{d}_{\mathrm{B}}{ }^{3}>/ 6$ are the magnetizations and the average volumes of the A and B particles, respectively (Fu et al., 2012).

Since the initial susceptibility is sensitive to the influence of the particle aggregates and the formation of clusters will lead to a larger initial susceptibility, whether or not there is any magnetic interaction between the magnetic colloidal particles in ferrofluids can be clarified by measurement of the initial susceptibility. Fu et al. (2012) investigated the initial susceptibility of binary ferrofluids based on $\gamma-\mathrm{Fe}_{2} \mathrm{O}_{3}$ and $\mathrm{ZnFe}_{2} \mathrm{O}_{4}$ nanoparticles. For the $\gamma-\mathrm{Fe}_{2} \mathrm{O}_{3}$ and $\mathrm{ZnFe}_{2} \mathrm{O}_{4}$ nanoparticles, the magnetization and morphology are shown in Fig. 7. The dipole coupling constants between $\gamma-\mathrm{Fe}_{2} \mathrm{O}_{3}$ particles $\lambda_{\gamma-\gamma}$, between $\mathrm{ZnFe}_{2} \mathrm{O}_{4}$ particles $\lambda_{\mathrm{Zn}-\mathrm{Zn}}$ and between $\gamma-\mathrm{Fe}_{2} \mathrm{O}_{3}$ and $\mathrm{ZnFe}_{2} \mathrm{O}_{4}$ particles $\lambda_{\text {y-Zn }}$, were calculated to be: $\lambda_{\gamma}$ $\gamma=1.28, \quad \lambda_{\mathrm{Zn}-\mathrm{Zn}}=1.39 \times 10^{-2}$ and $\lambda_{\gamma-\mathrm{Zn}}=7.30 \times 10^{-2}$, respectively. It is therefore clear that in the $\gamma-\mathrm{Fe}_{2} \mathrm{O}_{3}$ $\mathrm{ZnFe}_{2} \mathrm{O}_{4}$ binary ferrofluids, only $\gamma-\mathrm{Fe}_{2} \mathrm{O}_{3}$ nanoparticles can form aggregates by magnetic interaction. In these binary ferrofluids, $\phi_{\gamma}$ is fixed as $0.4 \%$ and the $\phi_{\mathrm{Zn}}$ are $0.1 \%$ (for sample (1)), $0.2 \%$ (for sample (2)) and $0.4 \%$ (for sample (3)). The magnetization curves of the binary ferrofluids are shown in Fig. 8. Since, for the ferrofluids, the influence of the particle interactions on the magnetic behaviors is most evident at weak field, the initial magnetization curves of the binary ferrofluids in the low field region $(\mathrm{H} \leq 5 \mathrm{kA} / \mathrm{m})$ are shown in the inset in Fig. 8. As a comparison, the magnetization curve of the $\gamma-\mathrm{Fe}_{2} \mathrm{O}_{3}$ nanoparticles multiplied by $0.4 \%$ is also given in Fig. 8 . 


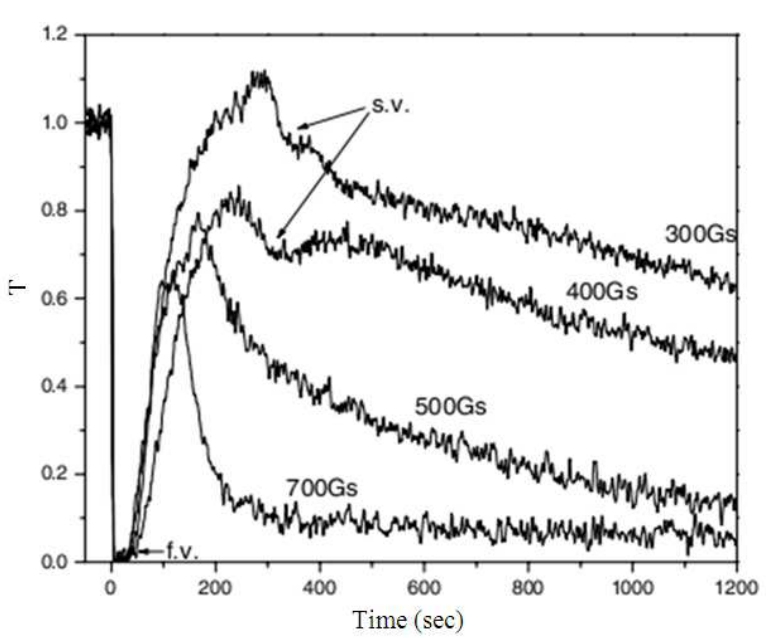

(a)



(b)

Fig. 9: The T-t curves, for different values of the magnetic field, of (a) $\mathrm{CoFe}_{2} \mathrm{O}_{4}$ ferrofluids with $\phi=0.6 \%$ and (b) $\mathrm{CoFe}_{2} \mathrm{O}_{4}-\mathrm{p}-\mathrm{MgFe}_{2} \mathrm{O}_{4}$ binary ferrofluids with $\phi_{\mathrm{AB}}=0.6 \%\left(\phi_{\mathrm{A}}=\phi_{\mathrm{B}}=0.3 \%\right)$ and p- $\mathrm{MgFe}_{2} \mathrm{O}_{4}$ ferrofluids with $\phi=0.6 \%$ in the inset (f. v., first valley; s.v., second valley). The magnetic field is applied at $\mathrm{t}=0$ (Li et al., 2011)

Table 1: The initial susceptibilities of the binary ferrofluids based on $\gamma-\mathrm{Fe}_{2} \mathrm{O}_{3} / \mathrm{ZnFe}_{2} \mathrm{O}_{4}$ nanoparticles multiplied by the volume fraction of the particles (Fu et al., 2012)

\begin{tabular}{llllc}
\hline$\phi_{\mathrm{Zn}}(\%)$ & 0 & 0.1 & 0.2 & 0.4 \\
\hline$\chi_{\mathrm{i}}$ of the binary ferrofluids & & 0.0563 & 0.0584 & 0.0401 \\
$0.4 \% \mathrm{f}_{\mathrm{g}}+\mathrm{f}_{\mathrm{Zn}} \mathrm{c}_{\mathrm{Zn}}$ & 0.0213 & 0.0215 & 0.0217 & 0.0220 \\
\hline
\end{tabular}

The initial susceptibilities of the binary ferrofluids and the particle system multiplied by the volume fraction of the particle are listed in Table1. From these experimental results, it can be seen that the initial susceptibility of the binary ferrofluids with $\phi_{\mathrm{Zn}}=0.4 \%$ is the smallest, although that with $\phi_{\mathrm{Zn}}=0.2 \%$ is larger than the one with $\lambda_{\mathrm{Zn}}=0.1 \%$. In addition, at high field, the magnetization of the ferrofluids with $\lambda_{\mathrm{Zn}}=0.4 \%$ is greatest. These results show that the formation of both chain-like and ring-like aggregates of $\gamma-\mathrm{Fe}_{2} \mathrm{O}_{3}$ nanoparticles can be stimulated by the $\mathrm{ZnFe}_{2} \mathrm{O}_{4}$ nanoparticle system. For dilute binary ferrofluids based on both strongly magnetic $\gamma-\mathrm{Fe}_{2} \mathrm{O}_{3}$ and weakly magnetic $\mathrm{ZnFe}_{2} \mathrm{O}_{4}$ nanoparticles, the microstructure and magnetization result mainly from the $\gamma-\mathrm{Fe}_{2} \mathrm{O}_{3}$ nanoparticle system, although the weakly magnetic $\mathrm{ZnFe}_{2} \mathrm{O}_{4}$ nanoparticle system modifies the microstructure and apparent magnetization behavior. These modifications affect the formation of chain-like and ring-like structures in the zero magnetic field. The former enhances the magnetization and leads to a large initial susceptibility and the latter reduces the magnetization at weak field to decrease the initial susceptibility. Therefore, the apparent initial susceptibility is a result of competing effects of both chain-like structures and ring-like structures on the magnetic behavior. The ratio of these aggregates varies non-monotonically with $\phi_{\mathrm{Zn}}$, so as $\phi_{\mathrm{Zn}}$ increases, the weakly magnetic $\mathrm{ZnFe}_{2} \mathrm{O}_{4}$ nanoparticle system produces a non-monotonic modification of the initial susceptibility of these binary ferrofluids.

The magneto-optical effects are very sensitive tools to determine the microstructure of the ferrofluids. When light is transmitted through binary ferrofluids composed of both ferrimagnetic $\mathrm{CoFe}_{2} \mathrm{O}_{4}$ nanoparticles and paramagnetic $\mathrm{p}-\mathrm{MgFe}_{2} \mathrm{O}_{4}$ particles in the presence of a high magnetic field and through pure (single) $\mathrm{CoFe}_{2} \mathrm{O}_{4}$ fluids in a low magnetic field, an oscillatory relaxation process is observed. This appears as two valleys in the $\mathrm{T}-\mathrm{t}$ curve for the variation of the relative transmission coefficient $\mathrm{T}$ with time $\mathrm{t}$ during the application of a magnetic field (Li et al., 2011), as shown in Fig. 9. This relaxation behavior is explained using a model of a bidispersed system based on both chained and unchained particles, in which, when an external magnetic field is applied, the chained particle will form chain like structures and the unchained particles behave as a magnetically polarized gas. In such a bidispersed system, the variation of the transmitted light results mainly from the motion of the chains, with the gas of magnetically polarized unchained particles producing the modulation effect. This oscillatory relaxation phenomenon depends on features of both the chained and unchained particles. If 
either of the particle volume fraction of chained particles or of unchained particles is very low, or the degree of polarization of the unchained particles is very weak, a simple nonlinear relaxation process will appear, giving only a valley in the T-t curves. For pure $\mathrm{CoFe}_{2} \mathrm{O}_{4}$ ferrofluids, the number of chained and unchained particles does not remain constant under different strengths of the applied magnetic field. With an increase of the magnetic field, some of the larger particles in the system of unchained particles will behave as chained particles and contribute to the chain-like structure. From an analysis of the relaxation behavior of the transmitted light, it is known that a binary ferrofluid based on strongly magnetic $\mathrm{CoFe}_{2} \mathrm{O}_{4}$ particles and weakly magnetic p$\mathrm{MgFe}_{2} \mathrm{O}_{4}$ particles, in which both chained and unchained particles can be regarded as roughly constant, is much closer to the theoretically bidispersed system than the pure ferrofluids containing only strongly magnetic $\mathrm{CoFe}_{2} \mathrm{O}_{4}$ nanoparticles.

In addition, binary ferrofluids based on two types of strongly magnetic nanoparticles can exhibit novel behavior. In the zero magnetic field, ferrofluids are isotropic colloidal suspensions of ferromagnetic or ferrimagnetic nanoparticles in a carrier liquid. When a magnetic field is applied to ferrofluids in the direction of the transmitted light, two longitudinal magnetooptical effects occur, circular birefringence (proportional to the Faraday Rotation (FR) and Circular Dichroism (CD). In most applications where longitudinal magneto-optical properties of materials are used (the optical isolator, for example), the FR is the useful effect whereas the $\mathrm{CD}$ generally induces performance limitations. The CD of ferrofluids is often large and generally cannot be neglected compared with the FR. For a Faraday rotator to be useful for polarization studies, it must be free of $\mathrm{CD}$ at the working wavelength. Donatini et al. (1997) have proposed a method for realizing, by mixing ferrofluids, a pure Faraday rotator (free of circular dichroism). In this method, they demonstrated that compatible ferrofluids, such as $\mathrm{Fe}_{3} \mathrm{O}_{4}$ and $\mathrm{CoFe}_{2} \mathrm{O}_{4}$, can be mixed in suitable proportions so as to cancel the $\mathrm{CD}$ at selected wavelengths in the visible-near-IR spectrum. Moreover, ferrofluids represent liquid materials that exhibit no residual birefringence, in contrast to most solid optical materials.

The binary ferrofluids based on micro-nano particles: Conventional ferrofluids consist of strongly magnetic ferromagnetic/ferrimagnetic nanoparticles dispersed in the liquid phase. In addition, for binary ferrofluids based on nano-nanoparticles, the dispersed phases consist of both strongly magnetic and weakly magnetic (e.g., paramagnetic) nanoparticles. Practically and already some time ago, some binary mixtures based on ferrofluids and micro-particles have been investigated experimentally. These can be viewed as ferrofluid composites (Charles, 1988) but they exhibit different behavior to single ferrofluids.It has been demonstrated that the use of ferrofluids as a carrier medium is an effective way of reducing the sedimentation of microsized particles. Therefore, the composite ferrofluid in which one is a ferrofluid consisting of magnetic nanoparticles and the other is a fluid consisting of magnetic or nonmagnetic microparticles, may have novel applications. Corresponding to the binary ferrofluids based on nanonano particles, such composites of ferrofluids can be viewed as binary ferrofluids based on micro-nano particles. Pater investigated the mechanism of chain formation in a binary mixture based on ferrofluid (nanofluid) and Magneto-Rheological (MR) fluid (microfluid) (Patel and Chudasama, 2009; Patel, 2011). In this bidispersed system based on both nanofluids and microfluids, in which the particles are from the same $\mathrm{Fe}_{3} \mathrm{O}_{4}$ family, the coupling parameter $\lambda$ for the micron size particles is very much larger $(\sim 100)$ than that of the nanomagnetic particles (1.3), which suggests that the dipolar interaction between the micron size magnetic particles is much stronger than that for the nanomagnetic particles. At high intensity of interparticle dipole-dipole interaction, the ferroparticles combine into short chains, whose number and length grow with an increase in the concentration and strength of the external magnetic field. For this reason, there is a possibility of large aggregation of microsized magnetic particles under the influence of a magnetic field, which leads to phase separation. However, when the nanomagnetic particles are mixed with micron size particles, the homogenous distribution of small particles changes the effective magnetic permeability of the background for the large particles, which reduces the effective dipolar interaction between the large particles. Hence, some of the nanomagnetic particles may become attached to the ends of the chains formed by the large particles, or fill the microcavities, as shown in Fig. 10. Thus, nanomagnetic particles of ferrofluids prevent larger aggregations of micron-size magnetic particles, so that the nanofluid based MR fluids are more stable than conventional MR fluid.

When the dipolar interactions are sufficiently strong, ferrofluids exhibit a rich phase behavior as a function of external field and volume fraction. The high-field solid-like phases have rheological properties that differ significantly from those of the low-field 
homogenous phases and large changes in system response and rigidity can be produced with relatively modest changes in the magnetic field. These switching properties have led to useful applications for ferrofluids, from sealants in the rotary shafts of computer disk drives to heat dissipaters in speaker coils. These demonstrated applications together with fundamental questions about dipolar liquids and solids have driven extensive theoretical and experimental investigation of the phase behavior of ferrofluids. Islarm and co-workers have explored the field induced phase transitions in aqueous ferrofluids and in aqueous mixtures of ferrofluids and nonmagnetic latex spheres (Islam et al., 2003). For the ferrofluid system, an isotropic to columnar phase transition was observed but no phase transition to a lamellar phase was observed. When non-magnetic particles of compatible and larger diameters were added to the ferrofluids, the resulting mixtures exhibited isotropic-columnar-lamellar phase transitions at much lower fields. Moreover, the columnar and the lamellar spacings decrease with increasing non-magnetic particle volume fraction and with increasing non-magnetic particle diameter. The qualitative and quantitative deviations between theory and experiment are not well understood.

Light is one of the most important and versatile phenomena in nature. Like a courier, it can transfer information from one point to another. Like an alchemist, it can alter matter. The interaction of photons with matter provides fascinating avenues for basic as well as applied research. Electromagnetism is the fundamental mediator of all interactions in atomic and condensed matter physics; it is rare to see an entirely new electromagnetic phenomenon (Desai, 2007). However, Mehta et al. (2006a) observed that upon application of an external magnetic field to a dispersion of micro-sized $\mathrm{Fe}_{3} \mathrm{O}_{4}$ particles stably dispersed in a ferrofluid based on $\mathrm{Fe}_{3} \mathrm{O}_{4}$ nanoparticles, through which monochromatic, coherent light was passing, the light gets trapped inside the suspension for a critical value of an applied magnetic field, which depends on the concentration of the ferrofluid and on the volume of the magnetic spheres (Mehta et al., 2006b). The photons remain in the trapped state as long as the external magnetic field is applied. A more complex and mysterious phenomenon is observed when switching off the magnetic field. When the field is removed, photons are emitted from the mixture in a preferred direction with the same frequency as that of the incident light, but with lower intensity. Mehta et al. (2006b) have proposed a qualitative explanation for the observed effect.
Table 2: Dependence of MSBG on particle size and incident light wavelength (Patel and Mehta, 2010)

\begin{tabular}{llrrr}
\hline $\begin{array}{l}\text { Size } \\
(\mu \mathrm{m}) \\
\left.{ }^{4} \mathrm{~T}\right)\end{array}$ & $\begin{array}{l}\text { Wavelength } \\
(\mathrm{nm})\end{array}$ & $\begin{array}{l}\mathrm{H}_{\mathrm{C} 1} \\
\left(\times 10^{-4} \mathrm{~T}\right)\end{array}$ & $\begin{array}{l}\mathrm{H}_{\mathrm{C} 2} \\
\left(\times 10^{-4} \mathrm{~T}\right)\end{array}$ & $\begin{array}{l}\Delta \mathrm{H} \\
\left(\times 10^{-}\right.\end{array}$ \\
\hline 3 & 780 & 80 & 160 & 80 \\
& 633 & 100 & 165 & 65 \\
& 532 & 150 & 195 & 45 \\
2 & 780 & 125 & 185 & 60 \\
& 633 & 160 & 205 & 45 \\
& 532 & 200 & 238 & 38 \\
0.5 & 780 & 180 & 210 & 30 \\
& 633 & 230 & 250 & 8 \\
& 532 & 300 & 408 & 8 \\
\hline
\end{tabular}

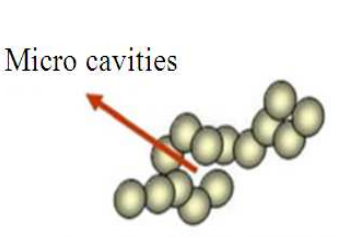

Nanoparticl
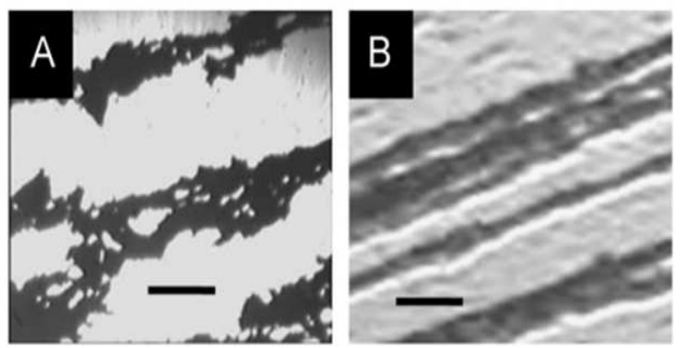

Fig. 10: Magnetically induced chain formation in (A) a conventional $\mathrm{Fe}_{3} \mathrm{O}_{4} \quad \mathrm{MR}$ fluid where microcavities are observed, (B) in a bidispersed MR fluid with $\mathrm{Fe}_{3} \mathrm{O}_{4}$ nanomagnetic particles. Nanoparticles fill the microcavities and are also attached to the end of the large particles (scale bar $5 \mu \mathrm{m})$ (Patel, 2011)
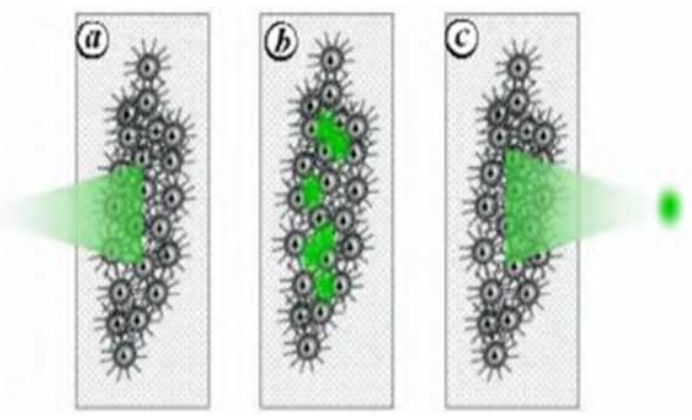

Fig. 11: Trapping of light in magnetic field-induced micro-cavities, (a) $\mathrm{H}=\mathrm{H}_{\mathrm{c}}, \mathrm{I}_{\mathrm{i}} \neq 0$ and $\mathrm{I}_{\mathrm{r}}=0$; (b) $\mathrm{H}=\mathrm{H}_{\mathrm{c}}, \mathrm{I}_{\mathrm{i}}=0$ and $\mathrm{I}_{\mathrm{r}}=0$; (c) $\mathrm{H}=0, \mathrm{I}_{\mathrm{i}}=0$ and $\mathrm{I}_{\mathrm{r}} \neq 0$ (Desai, 2007) 


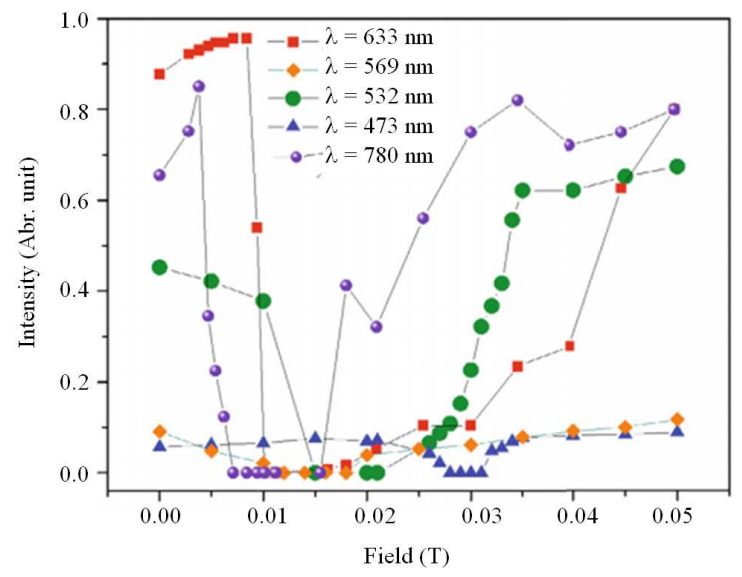

Fig. 12: The variation of light intensity emerging from the sample as a function of applied magnetic field for five different wavelengths. By decreasing the wavelength of the incident light, the stop band of the field appears at higher applied field (Patel and Mehta, 2010)

According to them, micron-sized magnetic spheres in the mixture of ferrofluids form elongated chain-like structures under the influence of the external magnetic field, in which Rayleigh scatterers (nanomagnetic particles in the ferrofluid) are arranged in a particular order. Thus, field-induced micro-cavities are formed in the suspension. When light enters these cavities, it will be homogeneously scattered in all directions within the cavity, as shown in Fig. 11. Micron-sized Mie scatterers (the magnetic spheres) keep the majority of the photons within the cavity by multiple scattering between the Mie and Rayleigh scatterers. A small number of photons may escape from the cavity by non-radiative means. Then, when the external magnetic field is removed, the micro-cavities will collapse in a preferred direction giving rise to the emission of photons in the forward and backward directions. The remarkable simplicity of the experiment, as well as that of the magnetic material used in the experiment, can lead to new potential applications in the field of optics and photonics.

Ge et al. $(2008 ; 2010)$ prepared superparamagnetic $\mathrm{Fe}_{3} \mathrm{O}_{4}$ colloidal nanocrystal clusters with high magnetization and high water dispersibility, which demonstrate excellent magneto-optical properties, including highly tunable stop bands and rapid and full reversibility over the entire visible spectrum. Patel and Mehta (2010) showed that it is possible to induce a Photonic Band Gap (PBG) at an optical frequency in dispersions of micron sized $\mathrm{Fe}_{3} \mathrm{O}_{4}$ particles in a ferrofluid based on $\mathrm{Fe}_{3} \mathrm{O}_{4}$ nanoparticles $(\sim 10 \mathrm{~nm})$ by subjecting it to a critical magnetic field, which is perpendicular to the electric vector of the incident light. Figure 12 shows the variation of light intensity emerging from the sample as a function of applied field for five different wavelengths. The sample contained $3 \mu \mathrm{m}$ diameter $\mathrm{Fe}_{3} \mathrm{O}_{4}$ particles stably dispersed in the ferrofluids. It is observed that for all the wavelengths, the intensity of the emergent light first decreases to zero upon increase of the field $\left(\mathrm{H}_{\mathrm{cl}}\right)$ and for certain regions of the field it remains zero. If the field is further increased $\left(\mathrm{H}_{\mathrm{c} 2}\right)$ then, again, the intensity increases. The region of the field $\left(\mathrm{H}_{\mathrm{c} 2}-\mathrm{H}_{\mathrm{c} 1}\right)$ within which the light remains inhibited is called the "Magnetic Stop Band Gap" (MSBG). Table 2 shows the values of the critical fields and the MSBG for three micron-sized particles for the five wavelengths. It can be seen from Fig. 12 and Table 2 that for all wavelengths except blue there is an overlap region of the MABG. If the applied magnetic field is within this overlap region then the corresponding wavelength will be inhibited. For example, if the field is $0.016 \mathrm{~T}$, then both the red and the green light should simultaneously disappear. This finding suggests that the system, consisting of micron-sized magnetic particles doped in a ferrofluid, performs as a photonic band gap material for visible polarized light that has its electric vector $\mathrm{E}$ perpendicular to the applied magnetic field with wavelengths between $\lambda=633 \mathrm{~nm}$ and $\lambda=532 \mathrm{~nm}$, i.e., a photonic band gap of $101 \mathrm{~nm}$. In other words, when white light is incident on the sample, it will reject waves having wavelengths between 633 and $532 \mathrm{~nm}$.

\section{CONCLUSION}

Ferrofluids, which are suspensions of magnetic nanoparticles in suitable carrier liquids, are not only of technological interest but they continue to be the object of much basic research. Except when they are very dilute, the effects of magnetic interactions in ferrofluids cannot be ignored, so that their magnetization behavior exhibits an essential deviation from the Langevin (super) paramagnetic theory. Real ferrofluids are more or less polydispersed, though size sorting can be performed in order to significantly reduce polydispersity (Lefebure $e t$ al., 1998; Yavuz et al., 2006). Even if small, the residual polydispersity is an awkward parameter as soon as the thermodynamic behavior of the system is discussed (Bartlett, 1997). For such ferrofluids, in which there is distribution of particle size, their properties (magnetic property, viscosity, magnetooptical effects and so on) are different from those of a monodispersed system. A few theoretical studies of bidispersed systems, consisting only of large and small 
particles, without any size distribution, have been presented. This bidispersed model of ferrofluids, consisting of both large and small particles, can be regarded as a system consisting of stronger and weaker magnetic particles with different magnetic moments $\mathrm{m}$, i.e., bidispersed magnetic dipolars fluids. Experimentally, one can synthesise a system based on two types of particles, with different magnetizations M, to simulate the bidispersed system. In other words, using the magnetically bidispersed system instead of the size bidispersed system, it is easier to discuss the properties of nanoparticle systems accurately by combining both experiments and theories. Experimental results show that such magnetically bidispersed ferrofluids, known as binary ferrofluids, could have different properties from the known ferrofluids and can be explored by the bidispersed model. In addition, experiments show that binary mixtures of both ferrofluids and microsized particles exhibit novel behaviors, although mechanisms for these are unclear at present. Consequently, binary ferrofluids based on two colloidal particles with different magnetizations will stimulate not only the field of ferrofluids but may also produce new physical phenomena and potential applications. We believe that both the binary ferrofluids based on nano-nano particles and those based on micro-nano particles can be integrated in a model of a bidispersed system based on aggregated and non-aggregated particles. With increasing magnetic field, the non-aggregated particles may transform into aggregated particles, i.e., the number of both non-aggregated and aggregated particles is not constant although the total number of particles is unchanged ( $\mathrm{Li}$ et al., 2011 ; 2012). Such a model, in which whether the numbers of aggregated and non-aggregated particles are conservative will be considered, could be helpful to advance the theory of ferrofluids. In summary, the synthesis and properties of binary ferrofluids based on two types particles, in which at least one is ferromagnetic or ferrimagnetic nanoparticles, are worthy of considerable further investigation.

\section{ACKNOWLEDGEMENT}

This study is supported by the Nature Science Foundation of China (11074205)

\section{REFERENCES}

Aoshima, M. and A. Satoh, 2005. Two-dimensional Monte Carlo simulations of a colloidal dispersion composed of polydisperse ferromagnetic particles in an applied magnetic field. J. Colloid Interf. Sci., 288: 475-488. DOI: 10.1016/j.jcis.2005.02.093
Bartlett, P., 1997. A geometrically-based mean-field theory of polydisperse hard-sphere mixtures. J. Chem. Phys., 107: 188-197. DOI: 10.1063/1.474364

Birdi, K.S., 1997. Handbook of Surface and Colloid Chemistry. 1st Edn., CRC Press, Boca Raton, ISBN-10: 0849394597, pp: 763

Buske, N., 1994. Application of magnetite sols in environmental technology. Prog. Collid. Polym. Sci., 95: 175-180. DOI: 10.1007/BFb0115720

Butter, K., P.H. Bomans, P.M. Frederik, G.J. Vroege and A.P. Phlipse, 2003. Direct observation of dipolar chains in iron ferrofluids by cryogenic electron microscopy. Nat. Matter, 2: 88-91. PMID: 12612691

Cabuil, V., 2000. Phase behavior of magnetic nanoparticles dispersions in bulk and confined geometries. Curr. Opin. Colloid Interf. Sci., 5: 4448. DOI: 10.1016/S1359-0294(00)00036-4

Cerdà, J.J., E. Elfimova, V. Ballengger, E. Kruikova, A. Ivanov and C. Holm, 2010. Behavior of bulky ferrofluids in the diluted low-coupling regime: Theory and simulation. Phys. Rev. E., 81: 011501. DOI: 10.1103/PhysRevE.81.011501

Charles, S.W., 1988. Aggregation in magnetic fluids and magnetic fluid composites. Chem. Eng. Comm., 67: 145-180. DOI: 10.1080/00986448808940382

Desai, J.N., 2007. Magnetic trapping of light. Curr. Sci., 93: 452-453.

Donatini, F., H. Sahash and J. Monin, 1997. Pure Faraday rotator: A ferrofluid mixing method. Appl. Opt. 36: 8165-8167. DOI: 10.1364/AO.36.008165

Dubois, E., V. Cabuil, F. Boue and R. Perzynski, 1999. Structural analogy between aqueous and oily magnetic fluids. J. Chem. Phys., 111: 7147-7160. DOI: $10.1063 / 1.480007$

Elfimova, E.A., A.O. Ivanov and P.J. Camp, 2012. Theory and simulation of anisotropic pair correlations in ferrofluids in magnetic fields. J. Chem. Phys., 136: 194502-194502. DOI: 10.1063/1.4717718, PMID: 22612098

Fu, J., J. Li, Y. Lin, X. Liu and L. Lin et al., 2012. The modification of the initial susceptibility of dilute binary ferrofluids based on $\gamma-\mathrm{Fe}_{2} \mathrm{O}_{3} / \mathrm{ZnFe}_{2} \mathrm{O}_{4}$ nanoparticles. Appl. Phys. A., 108: 155-160. DOI: 10.1007/s00339-012-6864-z

Ge, J., S. Kwon and Y. Lin, 2010. Niche applications of magnetically responsive photonic structures. J. Mater. Chem. DOI: 10.1039/c0jm00083c 
Ge, J., Y. Hu, T. Zhang, T. Huynh and Y. Yin, 2008. Self-assembly and field-responsive optical diffractions of superparamagnetic colloids. Langmuir, 24: $\quad 3671-3680 . \quad$ DOI: 10.1021/la7039493

Hamonay, E., J. Depeyrot, M. H. Sousa, F.A. Tourinho and J.C. Bacri et al., 1999. Optical properties of nickel ferrite ferrofluids. J. Magn. Magn. Mater., 201: 195-199. DOI: $10.1016 /$ S03048853(99)00023-2

Han, S., J. Li, R. Gao, T. Zhang and B. Wen, 2009. Study of magnetisation behaviours for binary ionic ferrofluids. J. Exp. Nanosci., 4: 9-19. DOI: 10.1080/17458080802688419

Holm, C. and J.J. Weis, 2005. The structure of ferrofluids: A status report. Curr. Opin. Colloid. Interf. Sci., 10: 133-140. DOI: 10.1016/j.cocis.2005.07.005

Holm, C., A. Ivanov, S. Kantorovich, E. Pyanzina and E. Reznikov, 2006. Equilibrium properties of a bidisperse ferrofluid with chain aggregates: Theory and computer simulations. J. Phys. Condens. Matter., 18: s2737-s2756. DOI: 10.1088/0953-8984/18/38/S14

Huke, B. and M. Lucke, 2004. Magnetic properties of colloidal suspensions of interacting magnetic particles. Rep. Prog. Phys., 67: 1731-1768. DOI: 10.1088/0034-4885/67/10/R01

Islam, M.F., K.H. Lin, D. Lacote, T.C. Lubensky and A.G. Yodh, 2003. Field-induced structures in miscible ferrofluid suspensions with and without latex spheres. Phys. Rev. E., 67: 021402. DOI: 10.1103/PhysRevE.67.021402

Ivanov, A.O. and O.B. Kuznetsova, 2001. Magnetic properties of dense ferrofluids: An influence of interparticle correlations. Phys. Rev. E., 64: 041405-041405.

DOI: 10.1103/PhysRevE.64.041405

Kantorovich, S.S., 2005. Chain aggregate structure in polydisperse ferrofluids: Different applications. J. Magn. Magn. Mater., 289: 203-206. DOI: 10.1016/j.jmmm.2004.11.059

Klokkenburg, M., C. Vonk, E.M. Claesson, J.D. Meeldijk and B.H. Erné et al., 2004. Direct imaging of zero-field dipolar structures in colloidal dispersions of synthetic magnetite. J. Am. Chem. Soc., 126: 16706-16707. DOI: 10.1021/ja0456252

Klokkenburg, M., R.P.A. Dullens, W.K. Kegel, B.H. Erné and A.P. Philipse, 2006. Quantitative realspace analysis of self-assembled structures of magnetic dipolar colloids. Phys. Rev. Lett., 96: 037203-037206.

DOI: 10.1103/PhysRevLett.96.037203
Lefebure, S., E. Dubois, V. Cabuil, S. Neveu and R. Massart, 1998. Monodisperse magnetic nanoparticles: Preparation and dispersion in water and oils. J. Mater. Res., 13: 2975-2981. DOI: 10.1557/JMR.1998.0407

Li, J., J. Fu, Y. Lin, X. Liu and L. Lin et al., 2012. The magneto-optical behaviors modulated by unaggregated system for $\gamma-\mathrm{Fe}_{2} \mathrm{O}_{3}-\mathrm{ZnFe}_{2} \mathrm{O}_{4}$ binary ferrofluids, AIP Adv., 2: 042124-042133. DOI: 10.1063/1.4765645

Li, J., X. Qiu, Y. Lin, X. Liu and J. Fu et al., 2011. Oscillatory-like relaxation behavior of light transmitted through ferrofluids. Appl. Opt., 50: 5780-5787. DOI: 10.1364/AO.50.005780

Li, J., Y. Huang, X. Liu, Y. Lin and L. Bai et al., 2007. Effect of aggregates on the magnetization property of ferrofluids: A model of gaslike compression. Sci. Tech. Adv. Mater., 8: 448-454. DOI: 10.1016/j.stam.2007.04.007

Li, J., Y. Lin, X. Liu, L. Lin and Q. Zhang et al., 2012. The quasi-magnetic-hysteresis behavior of polydisperse ferrofluids with small coupling constant. Physica B., 407: 4638-4642. DOI: 10.1016/j.physb.2012.08.033

Li, Q., J. Li, X. Chen, S. Han and R. Gao, 2008. Study of coercive force for $\mathrm{yZnFe}_{2} \mathrm{O}_{4}-(1-\mathrm{y}) \mathrm{CoFe}_{2} \mathrm{O}_{4}$ magnetic nanoparticles system. J. Exp. Nanosci., 3: 245-257. DOI: 10.1080/17458080802183635

Mehta, R.V., R. Patel and R.V. Upadhyay, 2006a. Direct observation of magnetically induced attenuation and enhancement of coherent backscattering of light. Phys. Rev. B., 74: 195127195125. DOI: 10.1103/PhysRevB.74.195127

Mehta, R.V., R. Patel, R. Desai, R.V. Upadhyay and K. Parekh, 2006b. Experimental evidence of zero forward scattering by magnetic sphere. Phys. Rev. Lett., 96: 127402-127405. DOI: 10.1103/PhysRevLett.96.127402

Mendelev, V. and A. Ivanov, 2005. Magnetic properties of ferrofluids: An influence of chain aggregates. J. Magn. Magn. Mater., 289: 211-214. DOI: 10.1016/j.jmmm.2004.11.061

Mérigue, G., M. Jardat and P. Turg, 2004. Structural properties of charge-stabilized ferrofluids under a magnetic field: A Brownian dynamics study. J. Chem. Phys., 121: 6078-6085. DOI: $10.1063 / 1.1784434$

Odenbach, S., 2003. Ferrofluids-magnetically controlled suspensions. Coll. Surf. A., 217: 171178. DOI: 10.1016/S0927-7757(02)00573-3 
Patel, R. and B. Chudasama, 2009. Hydrodynamics of chains in ferrofluid-based magnetorheological fluids under rotating magnetic field. Phys. Rev. E., 80: 012401-012404.

DOI: 10.1103/PhysRevE.80.012401

Patel, R. and R.V. Mehta, 2010. Experimental investigations on magnetically induced photonic band gap in ferrodispersions. Eur. Phys. J. Appl. Phys., 52: 30702-30707. DOI: 10.1051/epjap/2010152

Patel, R., 2011. Mechanism of chain formation in nanofluid based MR fluids. J. Magn. Magn. Mater., 323: 1360-1363. DOI: 10.1016/j.jmmm.2010.11.046

Phulé, P.P., 2001. Magnetorheological (MR) fluids: Principles and applications. Smart Mater. Bull., Feb.: 7-10. DOI: 10.1016/S1471-3918(01)80040$\mathrm{X}$

Psheichnikor, A.F. and V.V. Mekhonoshin, 2000. Equilibrium magnetization and microstructure of the system of superparamagnetic interacting particles: Numerical simulation. J. Magn. Magn. Mater., 213: 357-369. DOI: 10.1016/S03048853(99)00829-X

Taketomi, S. and R.D. Shull, 2002. Experimental study of magnetic interactions between colloidal particles in magnetic fluids. J. Appl. Phys., 91: 8546-8548. DOI: 10.1063/1.1452205

Taketomi, S. and R.D. Shull, 2003. Experimental verification of interactions between randomly distributed fine magnetic particles. J. Magn. Magn. Mater., 266: 207-214. DOI: 10.1016/S03048853(03)00478-5

Tavers, J.M., J.J. Weis and M.M.T. Gamm, 1999. Strongly dipolar fluids at low densities compared to living polymers. Phys. Rev. E., 59: 4388-4395. DOI: 10.1103/PhysRevE.59.4388

Teixeira, P.I.C., J.M. Tavares and M.M.T. Gama, 2000. The effect of dipolar forces on the structure and thermodynamics of classical fluids. J. Phys. Condens. Matter., 12: R411-R434. DOI: 10.1088/0953-8984/12/33/201
Wang, A.R., J. Li and R. Gao, 2009. The structural force arising from magnetic interactions in polydisperse ferrofluids. Appl. Phys. Lett., 94: 212501-212503. DOI: 10.1063/1.3141487

Wang, Z. and C. Holm, 2003. Structure and magnetic properties of polydisperse ferrofluids: A molecular dynamics study. Phys. Rev. E., 64: 041401041411. DOI: 10.1103/PhysRevE.68.041401

Wang, Z., C. Holm and H.W. Muller, 2002. Molecular dynamics study on the equilibrium magnetization properties and structure of ferrofluids. Phys. Rev. E., 66: 021405-021417. DOI: 10.1103/PhysRevE.66.021405

Yavuz, C.T., J.T. Mayo, W.W. Yu, A. Parkash and J.C. Falkner et al., 2006. Low-field magnetic separation of monodisperse $\mathrm{Fe}_{3} \mathrm{O}_{4}$ nanocrystals. Science, 314: 964-967. DOI: 10.1126/science. 1131475

Zhang, T.Z., J. Li, H. Miao, Q.M. Zhang and J. Fu et al., 2010. Enhancement of the field modulation of light transmission through films of binary ferrofluids. Phys. Rev. E., 82: 021403. DOI: 10.1103/PhysRevE.82.021403

Zubarev, A., 2002. Statistical physics of non-dilute ferrofluids. Odenbach, S., (Edn.), Ferrofluids, Magnetically Controllable Fluids and Their Application, 594: 143-161.

Zubarev, A.Y. and D.N. Chirkov, 2010. On the theory of the magnetoviscous effect in ferrofluids. J. Exp. Theor. Phys., 110: 995-1004. DOI: 10.1134/S1063776110060105

Zubarev, A.Y. and L.Y. Iskakova, 2003. Structural transformations in polydisperse ferrofluids. Colloid J. 65: 711-719. DOI: 10.1023/B:COLL.0000009113.62256.d8

Zubarev, A.Y., 2001. Rheological properties of polydisperse magnetic fluids. Effect of chain aggregates. J. Exp. Theor. Phys., 93: 80-88. DOI: $10.1134 / 1.1391522$ 